\title{
Investigating the effect of early bladder management in patients with chronic kidney disease and diabetes: Lessons learned from a failed pilot study
}

\author{
Luc A. Wittig'1 J. Matthew Andrews ${ }^{2}$; R. Trafford Crump ${ }^{3}$; Kevin V. Carlson ${ }^{4}$; Richard J. \\ Baverstock $^{4}$ \\ ${ }^{1}$ Department of Medicine, Cumming School of Medicine, University of Calgary, Calgary AB; ${ }^{2}$ Division of Urology, \\ Department of Surgery, Memorial University, St. John's, NL; ${ }^{3}$ Department of Surgery, Cumming School of \\ Medicine, University of Calgary, Calgary AB; ${ }^{4}$ Vesia [Alberta Bladder Centre]; Division of Urology, Department of \\ Surgery, University of Calgary, Calgary AB; Canada
}

Acknowledgement: Research supported by CUA/Pfizer Incontinence Fellowship

Cite as: Can Urol Assoc J 2018 May 28; Epub ahead of print.

http://dx.doi.org/10.5489/cuaj.5034

Published online May 28, 2018

***

The incidence of diabetes mellitus has increased worldwide, and is epidemic in Canada. As of 2015, it was estimated that 3.34 million (8.9\%) Canadians have diabetes.(1) Diabetes has potential to cause serious impairment of multiple organ systems, including neuropathy, retinopathy, and nephropathy. Diabetic autonomic neuropathy can lead to subsequent urological sequelae and profound effects on quality of life through erectile dysfunction, retrograde ejaculation, bladder dysfunction (diabetic cystopathy), and recurrent infections. Estimates range between $43-87 \%$ of type 1 diabetic patients and 25\% of type 2 diabetic patients develop bladder cystopathy.(2) Overactive bladder also appears to be more common in patients with diabetes.(3)

The worsening of chronic kidney disease (CKD) secondary to diabetic cystopathy is poorly understood. It has been well documented that patients with diabetes experience significantly more urinary conditions compared to their peers, including impaired bladder emptying, urinary tract infections (UTIs), and pyelonephritis.(4,5) These conditions in turn can potentially exacerbate CKD. Despite how common this condition is and the link to CKD, the benefits of early bladder management in these patients are poorly understood and no management guidelines are currently available.

We set out to address this gap in knowledge through a research study that was awarded the 2015 CUA-Pfizer Incontinence Fellowship Grant. Our hypothesis was that early management of lower urinary tract dysfunction in patients with CKD and diabetes both reduces morbidity associated with diabetic cystopathy [e.g. stabilization of glomerular filtration rates (GFR)], and increases patients’ quality-of-life (eg. via reduction in symptomatic UTIs). 
We designed a randomized, controlled trial to pilot test this hypothesis. We proposed to prospectively recruit a cohort of 50 diabetic patients over 18 years of age who were identified by nephrology as having CKD (stage 3-4 with GFR 15-59). Patients were to be randomized to an observation or treatment arm. Those within the treatment arm received renal and bladder diagnostic testing, and early urological management with the aim of optimizing bladder function. Where deemed necessary by urology, management options included any combination of urological medications or assisted bladder drainage. Urodynamic studies (UDS) were to be conducted at baseline and 1 year, and flow rates and bladder scans at 3 and 6 months. The observation arm would not receive urological intervention, and remained under the management of nephrology. Laboratory investigations were to be performed at regular intervals for both groups.

Unfortunately, over an 18-month period, our study was able to recruit only 14 of the desired 50 participants. Of the 14 recruited, 7 withdrew citing either: 1) no time, or 2) unable to make it to the clinic for the diagnostic testing. Despite an enthusiastic relationship with nephrology including joint rounds, email reminders, and a collaborative research team, we ultimately closed the study prior to reaching our sample size target.

The decision to publish our experience stems from an obligation we feel to our grant sponsors (CUA-Pfizer), and to our colleagues who may benefit in the future from our learnings. Despite an accepted protocol, ethics approval, and an enthusiastic and talented research support team, a number of factors specific to this study population likely lead to failure of this study to reach fruition.

Firstly, while enrollment always poses its challenges, this may be particularly true in the CKD population. Practitioners often feel that they have a "ton of these patients", but in reality this does not necessarily translate to enrollment or compliance with research protocols. As a whole CKD patients are reported to have a poor compliance with their health care regime and non-adherence to medication has been reported as high as $74 \%$ in some cases.(6) Furthermore, a recent study suggests that consent rates for interventional studies in CKD patients are only approximately 50\%.(7)

Patients with CKD may experience health care fatigue: Owing to multiple co-morbidities, they are typically followed by multiple care providers and are frequently attending appointments and having investigations done. It has been our experience that scheduling and attendance of additional appointments and/or investigations pertaining to a research protocol is met with marked challenges due to competing health appointments, diagnostic testing, and ongoing or new medical concerns. To the patient, these ongoing medical commitments may seem never-ending, and combined with physical symptoms of fatigue many CKD patients understandably experience a sense of being "overwhelmed".(8) This may lead to patients foregoing medical care appointments and education opportunities.(8) Understandably, the additional demands of participation in research may be perceived as a low priority. When asked, many of our patients 
expressed openness to participate in research, but could not commit to scheduled visits due to pre-existing medical commitments.

Finally, our protocol included urodynamics as we felt this would provide a more accurate assessment of bladder function. Anecdotally, we have observed that urodynamic testing is associated with some amount of patient fear and anxiety. This is in agreement with previous reports indicating urodynamic investigations provoke moderate anxiety in about half of survey respondents.(9) We have learned through this study experience that this minimally invasive test is often the final nail in the recruitment coffin as it may deter the casual participant, and frighten away even those patients most committed to research.

For any research group, it is critical to take stock of successes and failures. Despite the efforts of an experienced and collaborative team, our pilot study failed to enroll subjects and had to be terminated. We thus reflect upon our lessons learned such that others may gain from our experiences. Investigators choosing to wade into these waters in the future will need to address the aforementioned recruitment challenges in their efforts to maximize research success. 


\section{References}

1. Canadian Diabetes Association. 2015 Report on diabetes: Driving change. Toronto, ON: CDA; 2015.

2. Deli G, Bosnyak E, Pusch G, et al. Diabetic neuropathies: Diagnosis and management. Neuroendocrinology 2014;98:267-80.

3. Daneshgari F, Liu G, Birder L, et al. Diabetic bladder dysfunction: Current translational knowledge. J Urol 2009;182:S18-S26

4. Hirji I, Guo Z, Andersson SW, et al. Incidence of urinary tract infection among patients with type 2 diabetes in the UK General Practice Research Database (GPRD). J Diabetes Complications 2012;26:513-6.

5. Arrellano-Valdez F, Urrutia-Osorio M, Arroyo C, et al. A comprehensive review of urologic complications in patients with diabetes. Springerplus 2014;3:549.

6. Nielsen TM, Johl MF, Feldt-Rasmussen B, et al. Adherence to medication in patients with chronic kidney disease: a systematic review of qualitative research. Clin Kidney $\mathrm{J}$ 2017. Sfx140. https://doi.org/10.1093/ckj/sfx140

7. Selewski DT, Herreshoff EG, Gipson DS. Optimizing Enrollment of Patients into Nephrology research studies. Clin J Am Soc Nephrol 2016;11:512-7.

8. Narva AS, Norton JM, Boulware LE. Educating patients about CKD: The path to selfmanagement and patient-centered care. Clin J Am Soc Nephrol 2016;11:694-703

9. Gorton E, Stanton S. Womens attitude to urodynamics: a questionnaire survey. British $J$ Obstet Gynaecol 1999; 106:851-6. 